\title{
EDITORIAL
}

\section{Yes, It is Possible}

\author{
Gautam N. Allahbadia ${ }^{1}$
}

Published online: 13 November 2015

(C) Federation of Obstetric \& Gynecological Societies of India 2015

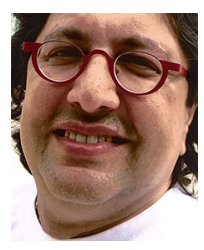

\begin{abstract}
About the Author
Gautam N Allahbadia, MD is the Editor-in-Chief of the Journal of Obstetrics and Gynecology of India as well as the IVF Lite (Journal of Minimal Stimulation IVF). He is the Medical Director of Rotunda-The Center for Human Reproduction, the world-renowned infertility clinic at Bandra, and Rotunda-Blue Fertility Clinic and Keyhole Surgery Center, Mumbai, India. $\mathrm{He}$ is a noted world authority on ultrasound-guided embryo transfers and one of the pioneers in Third Party Reproduction in Southeast Asia. Dr. Allahbadia was responsible for India's first trans-ethnic surrogate pregnancy involving a Chinese couple's baby delivered by an unrelated Indian surrogate mother. He cherishes over 125 peer-reviewed publications, 134 book chapters and 22 textbooks, the latest being a comprehensive text, entitled "Minimal Stimulation IVF", and is on the Editorial Board of several International Journals. He just completed a two year tenure as Medical Director at the New Hope IVF Clinic, Sharjah, UAE, where he introduced IVF Lite to the country. Dr. Allahbadia has recently been elected as the Vice President of the World Association of Reproductive Medicine (WARM), headquartered in Rome, and "Mumbai's Top Doc" for 2012 by a peer nomination
\end{abstract} process. You can read more about his work at www.gautamallahbadia.com.

\begin{abstract}
Human immunodeficiency virus-serodiscordant couples are actively seeking reproductive assistance and often consider or practice unsafe measures to achieve pregnancy. Reproductive issues and concerns unique to these couples need to be addressed before treatment.
\end{abstract}

Gautam N Allahbadia is the Editor-in-Chief of the Journal of Obstetrics and Gynecology of India as well as the IVF Lite (Journal of Minimal Stimulation IVF) and Medical Director of Rotunda-The Center for Human Reproduction, Bandra, and Rotunda-Blue Fertility Clinic and Keyhole Surgery Center, Parel, Mumbai, India.

Gautam N. Allahbadia

ivfwaladoc@gmail.com

1 Rotunda-The Center For Human Reproduction, Mumbai, India
Assisted reproduction techniques can minimize the risk of infection and complete families associated with serodiscordant couples. Since 1987, more than 4000 published attempts have been reported in which processed spermatozoa from HIV-seropositive men were used to establish pregnancy in HIV-seronegative women. When the female partner is HIV positive, intrauterine insemination (IUI) will suffice in order to prevent horizontal infection. However, when the male partner is HIV positive, a technique developed in Milan over 15 years ago, involving sperm washing, is used in order to minimize infection of the healthy partner. Some couples need further treatment, due to inherent infertility, with advanced reproductive technology (ART) procedures, such as IVF or ICSI. Recent innovative approaches such as pre-exposure prophylaxis 
(PrEP) with antiretroviral drugs may reduce further the susceptibility of the uninfected female partner. Numerous ART centers worldwide treat these couples. Most centers are equipped with separate laboratory space for collecting specimens from infected patients and provide separate storage tanks for freezing infected gametes and embryos in order to protect other patients using the facility. There are no reports of HIV infection of laboratory personnel resulting from processing the gametes/embryos for serodiscordant couples using current laboratory protocols. Cross-contamination of the gametes or embryos of other couples in the same laboratory has also not been reported. The risk is theoretical only, particularly when standard universal precautions are used.

Keywords Human immunodeficiency virusserodiscordant couples · HIV - Transmission of virus . Hepatitis C Virus

\section{Introduction}

The HIV transmission rate to an uninfected partner is estimated to be approximately 1 in 500-1000 episodes of unprotected intercourse [1]. The risk of viral transmission increases dramatically if the HIV-infected partner's viral load is high or if the HIV-uninfected partner has a concomitant genital infection, inflammation, or abrasions [1]. Increasingly, HIV-1-seropositive individuals are living active and productive lives as a result of highly active antiretroviral therapy (HAART). Most infected patients are of reproductive age and would normally desire to begin families. During the past 15 years, assisted reproductive techniques (ART) have been introduced to reduce or eliminate the infectious material known to exist in semen [2]. Several European clinics and a few Indian centers offer assistance to HIV-serodiscordant couples in order to prevent infection in the seronegative partner. Treatments involve seropositive men and seronegative women undergoing either intrauterine insemination or in vitro fertilization (IVF). Marina et al. [3], as early as 1998, reported on use of semen from men who were seropositive for human immunodeficiency virus type 1 (HIV-1) to inseminate their partners without infecting them. The men provided 107 semen samples that were prepared with the use of the Percoll and swim-up techniques. The presence of HIV-1 was determined in the fraction of motile spermatozoa obtained after washing. If HIV-1 was not detected, IUI was performed in stimulated cycles. Human immunodeficiency virus type 1 RNA and DNA were detected with the use of the polymerase chain reaction techniques modified for spermatozoa. One hundred and seven semen samples were washed. Human immunodeficiency virus type 1 was not detected in 101 samples $(94.4 \%)$ and was detected in 6 samples $(5.6 \%)$. In the latter cases, IUI was not performed. One hundred and one IUI procedures were performed in 63 women. Thirty-one pregnancies resulted, for a pregnancy rate of $30.7 \%$ per cycle and $49.2 \%$ per inseminated woman. Thirty-seven healthy children were born. The results of tests for the detection of HIV-1 and antibodies to HIV-1 in the inseminated women were negative. The publication concluded that on the basis of the results, testing for HIV-1 with the use of the polymerase chain reaction technique on the semen fraction obtained after washing appears to prevent infection in the inseminated woman. This method makes it possible to help HIV-1-seropositive men to have children without infecting their female partners [3].

\section{Discussion}

Pasquier et al. [4] assessed the isolation process for motile spermatozoa for reducing the transmission of HIV and hepatitis $\mathrm{C}$ virus ( $\mathrm{HCV}$ ) during artificial insemination in HIV-serodiscordant couples in which the man is infected. Thirty-two HIV-1-infected clinically asymptomatic men have a median CD4 cell count of $396 \times 10(6) / 1$ and a median blood plasma HIV-1 RNA content of 414 copies/ ml. Of these, 16 were infected with both HIV and HCV. Motile spermatozoa were isolated from 51 semen samples by density gradient and 'swim-up.' HIV-1 and HCV genomes were detected and quantified in the blood plasma and seminal plasma and detected in seminal cell fractions obtained during spermatozoa isolation. HIV-1 RNA was detected in $30 \%$ of seminal plasma samples. HIV-1 genomes were found in $18 \%$ of seminal cell samples, but in none of the motile spermatozoa fractions after 'swim-up.' There was no correlation between the HIV-1 RNA concentrations in the blood and seminal plasma. HIV-1 genome was detected intermittently in patients who gave more than one sample. HCV RNA was detected in $20 \%$ of seminal plasma samples from HCV viraemic patients, but in no seminal cells or motile spermatozoa fractions. Purification of motile spermatozoa by density gradient plus 'swim-up' reduced the HIV-1 and HCV genomes in the semen of infected individuals to undetectable levels [4]. This method, associated with a standardized virus assay, could be useful for serodiscordant couples (males infected) who wish to have children [4].

Two hundred and thirteen IUIs were performed by Bujan et al. [5] in 56 HIV-1-serodiscordant couples, and 37 pregnancies occurred, resulting in 33 births and 2 ongoing pregnancies. Fifty percent of couples had a child. No women were infected. Their study demonstrated the efficiency and safety of an IUI program with prepared and virologically tested spermatozoa in serodiscordant couples 
[5]. At the Academic Medical Centre, Amsterdam, 20 serodiscordant couples underwent 76 IUI cycles [6]. An insemination was performed in 50 cycles $(66 \%)$. The insemination was cancelled in 26 cycles, because of too many follicles (risk of multiple pregnancy), weekend (no possibilities for virological testing), not enough spermatozoa after preparation, a positive HIV-1 RNA test and other reasons. Ten out of 20 women became pregnant $(50 \%)$, and 8 women were ongoing pregnant. The clinical and ongoing pregnancy rate per started cycle was 13 and $11 \%$, respectively. None of the mothers or babies seroconverted within the study period [6].

The first pregnancy achieved in a seronegative woman following in vitro fecundation through intracytoplasmic sperm (ICSI) injection from a man with autoimmune deficiency syndrome (AIDS; HIV-1 carrier) was reported in 1998 [7]. The semen was prepared by PureSperm and swim-up techniques. Some of the motile spermatozoa obtained were used to detect the presence of HIV-1 using the polymerase chain reaction technique. HIV-1 in DNA or RNA form was not detected using this technique. The remaining spermatozoa were frozen. Ovarian stimulation in the woman was performed with long-protocol analogues and gonadotropins. Thirteen mature oocytes were recovered, into which the thawed spermatozoa were microinjected. Nine embryos were obtained. Four were frozen, four transferred and one discarded. The woman became pregnant. Analyses for HIV-1 in the woman, performed in the first and third months of pregnancy, gave negative results. This case provided further experience for this private practice, with washed semen of sufficient quality for performing artificial insemination in HIV-1-serodiscordant couples (101 inseminations, 31 pregnancies, 28 deliveries, 37 babies, all healthy) [7]. In women with obstructed fallopian tubes, or when the semen is not of sufficient quality for artificial insemination techniques to be performed, ICSI can be carried out using frozen, HIV-1-free semen [7].

Ohl et al. [8] assessed the efficacy of ART in 57 couples in which at least one partner had human immunodeficiency virus (HIV-1) infection that was currently under control (47 men and 10 women). The semen of seropositive men was prepared and tested for viruses. Assisted reproduction techniques included intrauterine insemination (IUI), IVF and ICSI. For couples with seropositive men, five IUI and 49 IVF or ICSI attempts were preformed, while for seropositive women, these numbers were three IUI and 12 IVF or ICSI. No pregnancy occurred following the eight IUI trials. Seroconversion was not observed in any partners of seropositive men. Efficacy of treatment for these couples with ICSI was good, and the clinical pregnancy rate per embryo transfer was $48.8 \%$. The results for seropositive women were disappointing, with a clinical pregnancy rate per embryo transfer of $9.1 \%$ [8].
Seven hundred and forty-one couples entered Savasi et al.'s study of a possible 2011 serodiscordant couples counseled over 4 years [9]. Superovulation and IUI were performed in 581 couples, where the pregnancy rate per cycle and pregnancy rate per couple were 19 and $78 \%$, respectively, with multiple pregnancy rate being $4 \%$. One hundred and sixty couples were treated by IVF/ICSI, where pregnancy rate per cycle and per couple were 22 and $41 \%$, respectively, with multiple pregnancy rate being $10 \%$. All female partners were still HIV-1 negative at follow-up. The overall pregnancy rate $(70.3 \%)$, independent of the procedure used (IUI or IVF/ICSI), justifies the effort of the medical team in setting up and implementing dedicated centers and of the individual patient in seeking a safe pregnancy [9].

A woman preparing to undergo IVF and intracytoplasmic sperm injection to avoid horizontal viral transmission of HIV from her seropositive husband was discovered to be HIV seropositive, presumably secondary to a condom break or unprotected intercourse [10]. Had this event occurred after treatment, the sperm-washing technique used to avoid infection would have undoubtedly been called into question.

Vitorino et al. published a systematic review of five databases of noncomparative open intervention and observational studies of serodiscordant couples undergoing ART, with study selection and data extraction performed independently and in duplicate. Of the 658 abstracts retrieved, 41 were selected for review, and 17 full articles were included (3900 IUI cycles in 1184 couples in 11 aggregated studies and 738 ICSI/IVF cycles in 579 couples across 10 studies). The IUI and ICSI results were, respectively, pregnancy rates per cycle, 18 and $38.1 \%$; cumulative pregnancy, 50 and $52.9 \%$; and abortion rate, 15.6 and $20.6 \%$. No seroconversions in women or newborns were detectable at birth or after 3-6 months. Cumulative evidence suggests that ART is safe and effective for avoiding horizontal and vertical transmission in HIVserodiscordant couples [11].

In 2014, a published systematic review and meta-analysis included 24 studies with extractable data for HIVserodiscordant couples undergoing intrauterine insemination (IUI) or in vitro fertilization (IVF) [12]. For serodiscordant couples, HIV-positive men or women undergoing IUI and IVF treatment had a 17, 30, 14 and $16 \%$ per cycle fecundability, respectively. Multiple gestation rates were $10,33,14$ and $29 \%$, respectively. Miscarriage rates were $19,25,13$ and $20 \%$, respectively. No HIV transmission was observed in 8212 IUI and 1254 IVF cycles, resulting in $95 \%$ confidence that the true rate is 4.5 transmissions per 10,000 IUI cycles or less [12].

Lack of apparent transmission of HIV to partner or child with sperm washing and intrauterine insemination (IUI) or 
with IVF with ICSI has been reported for discordant (male positive) couples. Highly active antiretroviral therapy can reduce the viral burden in a person's serum and semen. Some centers have also adopted testing of sperm by using a polymerase chain reaction (PCR) assay to determine whether the virus is present in the washed sperm preparation, but the utility and effectiveness of this added step have been questioned by other centers that have eliminated PCR from their protocols [13-17]. Recent innovative approaches such as pre-exposure prophylaxis (PrEP) with antiretroviral drugs may reduce further the susceptibility of the uninfected female partner [14, 18].

If a woman is infected with HIV and her male partner is uninfected, transmission of infection to the male partner can be avoided by using homologous insemination with the partner's sperm. If this option is not available to the couple, or for other reasons not desired, there are considerable data showing that the risk of transmission can be minimized by using timed intercourse, assuring that the woman's viral load is suppressed to undetectable levels on antiretroviral therapy and/or that the uninfected male is taking antiretroviral therapy as PrEP [14]. While clinicians would need to emphasize that this option is not as safe as homologous insemination, it does represent an alternative option for select couples. More recent data demonstrate that combination antiretroviral treatment given to HIV-infected women antenatally further reduces transmission to offspring to $<2 \%$ [19].

\section{Recent Advances}

Antiretroviral therapy (ARV) for the infected partner and pre-exposure prophylaxis (PrEP) for the uninfected partner reduce the risk of heterosexual HIV transmission. Several demonstration projects suggest the feasibility and acceptability of antiretroviral (ARV)s as periconception HIV prevention for HIV-serodiscordant couples [19]. The application of ARVs to periconception risk reduction may be limited by adherence. For male-infected $(\mathrm{M}+\mathrm{F}-)$ couples who cannot access sperm processing and femaleinfected $(\mathrm{F}+\mathrm{M}-)$ couples unwilling to carry out insemination without intercourse, ARV for the infected partner, PrEP for the uninfected partner, combined with treatment for sexually transmitted infections, sex limited to peak fertility and medical male circumcision (for F + M- couples), provide excellent, well-tolerated options for reducing the risk of periconception HIV sexual transmission (XX). More recent research is investigating the efficacy of PrEP in which the uninfected female partner is treated with antiretroviral therapy during the time conception is attempted. In one study of 46 serodiscordant couples in which the female was treated with oral tenofovir, none of the women became infected with HIV and pregnancy rates reached $75 \%$ after 12 attempts 21 . This and other studies indicate that adherence to clinical protocols is essential for preventing transmission. The US Food and Drug Administration (FDA) has approved antiretroviral therapy for this use, although further research on the safety and efficacy of PrEP is ongoing [21]. Clinicians who elect to offer this therapy are encouraged to educate their patients about its potential risks and benefits as well as all available alternatives for safer conception.

\section{Conclusions}

It is important that physicians be aware of reproductive options available to couples affected by HIV and be prepared to engage in nonjudgmental dialog with patients. Couples where the male partner is HIV-infected but the female partner is not often express a desire for children. Serodiscordant couples in whom the man is HIV-1 positive and the woman is negative have limited options if they wish to have children safely, because sexual intercourse carries a 1-in-500 risk of transmitting virus in semen to the female partner. Sperm washing is a risk-reduction option in which infected sperm are washed free of virus before insemination into the female partner at the time of ovulation. Absence of detectable HIV is verified before insemination using a polymerase chain reaction nucleic acidbased sequence amplification assay. Pregnancy rate per insemination is $14 \%$, based on a European experience of more than 2000 inseminations; to date, there have been no seroconversions in either mother or child [22]. Washed sperm have also been used in other assisted conception treatments, such as in vitro fertilization. We suggest that sperm washing should only be carried out in dedicated units using a multidisciplinary approach to ensure that couples receive adequate pre-conceptional counseling, detailed sexual health and fertility assessment, and careful monitoring of the woman's HIV status during treatment and pregnancy. Evidence-based practice and social justice suggest that our field should increase access to care for HIV-serodiscordant couples.

\section{References}

1. Mandelbrot L, Heard I, Henrion-Geeant E, et al. Natural conception in HIV-negative women with HIV-infected partners. Lancet. 1997;349:850-1.

2. Coll O, Vidal R, Martinez de Tejada B, et al. Management of HIV serodiscordant couples. The clinician point of view. Contracept Fertil Sex. 1999;27(6):399-404.

3. Marina S, Marina F, Alcolea R, et al. Human immunodeficiency virus type 1 -serodiscordant couples can bear healthy children 
after undergoing intrauterine insemination. Fertil Steril. 1998;70(1):35-9.

4. Pasquier C, Daudin M, Righi L, et al. Sperm washing and virus nucleic acid detection to reduce HIV and hepatitis $\mathrm{C}$ virus transmission in serodiscordant couples wishing to have children. AIDS. 2000;14(14):2093-9.

5. Bujan L, Pasquier C, Labeyrie E, et al. Insemination with isolated and virologically tested spermatozoa is a safe way for human immunodeficiency type 1 virus-serodiscordant couples with an infected male partner to have a child. Fertil Steril. 2004;82(4):857-62.

6. van Leeuwen E, de Vries JW, Jurriaans S, et al. Intra uterine insemination with processed sperm for HIV serodiscordant couples in whom the man is HIV positive. Ned Tijdschr Geneeskd. 2005;149(8):423-4.

7. Marina S, Marina F, Alcolea R, et al. Pregnancy following intracytoplasmic sperm injection from an HIV-1-seropositive man. Hum Reprod. 1998;13(11):3247-9.

8. Ohl J, Partisani M, Wittemer C, et al. Assisted reproduction techniques for HIV serodiscordant couples: 18 months of experience. Hum Reprod. 2003;18(6):1244-9.

9. Savasi V, Ferrazzi E, Lanzani C, et al. Safety of sperm washing and ART outcome in $741 \mathrm{HIV}$-1-serodiscordant couples. Hum Reprod. 2006;22(3):772-7 Epub 2006 Nov 15.

10. Sauer MV, Choi J. HIV seroconversion in a woman preparing for assisted reproduction: an inherent risk in caring for HIVserodiscordant couples. Reprod Biomed Online. 2006;12(3):375-7.

11. Vitorino RL, Grinsztejn BG, deAndrade CA, et al. Systematic review of the effectiveness and safety of assisted reproduction techniques in couples serodiscordant for human immunodeficiency virus where the man is positive. Fertil Steril. 2011;95(5):1684-90. doi:10.1016/j.fertnstert.2011.01.127 Epub 2011 Feb 16.

12. Barnes A, Riche D, Mena L, et al. Efficacy and safety of intrauterine insemination and assisted reproductive technology in populations serodiscordant for human immunodeficiency virus: a systematic review and meta-analysis. Fertil Steril. 2014;102(2):424-34. doi:10.1016/j.fertnstert.2014.05.001 Epub 2014 Jun 18.
13. Sauer M. Sperm washing techniques address the fertility needs of HIV seropositive men: a clinical review. Reprod Biomed Online. 2005; 10:135-40.

14. HHS Panel on Treatment of HIV-Infected Pregnant Women and Prevention of Perinatal Transmission. Recommendations for use of antiretroviral drugs in pregnant HIV-1-infected women for maternal health and interventions to reduce perinatal HIV transmission in the United States. http://aidsinfo.nih. gov/contentfiles/ lvguidelines/PerinatalGL.pdf. Accessed 9 April 2015.

15. Kambin S, Batzer F. Assisted reproductive technology in HIV serodiscordant couples. Sex Reprod Menopause. 2004;2:92-100.

16. Sauer MV, Wang JG, Douglas NC, et al. Providing fertility care to men seropositive for human immunodeficiency virus: reviewing 10 years of experience and 420 consecutive cycles of in vitro fertilization and intracytoplasmic sperm injection. Fertil Steril. 2009;91:2455-60.

17. Peckham C, Newell ML. Preventing vertical transmission of HIV infection. N Engl J Med. 2000;343:1036-7.

18. Practice Committee of American Society for Reproductive Medicine. Recommendations for reducing the risk of viral transmission during fertility treatment with the use of autologous gametes: a committee opinion. Fertil Steril. 2013;99:340-6.

19. Centers for Disease Control and Prevention. Achievements in public health. Reduction in perinatal transmission of HIV infection-United States, 1985-2005. MMWR Morb Mortal Wkly Rep. 2006;55:592-7.

21. Matthews LT, Smit JA, Cu-Uvin S, et al. Antiretrovirals and safer conception for HIV-serodiscordant couples. Curr Opin HIV AIDS. 2012;7(6):569-78. doi:10.1097/COH.0b013e328358bac9.

22. Vernazza PL, Graf I, Sonnenberg-Schwan U, et al. Preexposure prophylaxis and timed intercourse for HIV-discordant couples willing to conceive a child. AIDS. 2011;25:2005-8.

23. Public Health Service; Preexposure Prophylaxis for the Prevention of HIV Infection in the United States-2014. http://www.cdc.gov/hiv/pdf/PrEPguidelines2014.pdf. Accessed 9 April 2015.

24. Gilling-Smith C. HIV prevention. Assisted reproduction in HIVdiscordant couples. AIDS Read. 2000;10(10):581-7. 